\title{
Felser, Axel (2019): Werte und Werthaltungen von Grundschullehrkräften
}

\author{
Münster und New York: Waxmann
}

\section{Alexandra Retkowski}

Online publiziert: 13. Januar 2021

(C) Der/die Autor(en) 2021

Die Auseinandersetzung mit und Analyse von Werten und Werthaltungen, normativen Orientierungen, moralischen Praxen und ethischen Diskursen ist ein kontinuierlicher, wenn auch kein zentraler Bestandteil der erziehungswissenschaftlichen Schul-, Schüler*innen- und Professionsforschung (vgl. etwa Benner 2009; Heinzel et al. 2017; Höhn 2003; Oser 1998). An diesen Fachdiskurs schließt Axel Felsers Studie zu Wertpräferenzen und Werthaltungen von Grundschullehrer*innen aus Bayern an. Der Grundschule ,als erste gemeinsame Schule für alle Kinder“ wird dabei im Spannungsverhältnis von Helmut Fends Enkulturations- sowie Sozialisationsfunktion und einer Orientierung an der Mündigkeit von Schüler*innen eine primäre Bedeutung zugemessen (Felser 2019, S. 56). „Werte zu thematisieren“ werde dabei im Fachdiskurs ,einerseits als Grundsatzaufgabe, andererseits auch als explizites Programm oder additiver Inhalt angesehen“ (S. 72). In jedem Fall fließen für Felser die fachlichen Begründungslinien der schulischen Wertediskussion in der individuellen Person der Lehrkraft zusammen - sei es durch die rechtlichen, fachlichen oder gesellschaftlichen Ansprüche an Lehrkräfte, die konkrete Umsetzung im Klassenzimmer gegenüber einer heterogenen Schüler*innenschaft oder auf Grund von biographischen, psychologischen und persönlichen Dispositionen (ebd.).

Die Studie arbeitet mit einem explanativen Methoden-Mix, welcher aus einer Fragebogenstudie mit 460 Grundschullehrer*innen sowie aus halbstrukturierten Leitfadeninterviews mit 20 aus der Fragebogenstichprobe gewonnenen Lehrkräften besteht (vgl. S. 103 ff.). Den nicht-zufälligen und nicht auf einer gezielten SamplingStrategie, sondern durch persönliche Kontaktaufnahme gewonnenen Untersuchungs-

Prof. Dr. A. Retkowski ( $\triangle)$

Campus Sachsendorf, Brandenburgische Technische Universität, Lipezker Straße 47, 03047 Cottbus, Deutschland

E-Mail: alexandra.retkowski@b-tu.de 
teilnehmer*innen des quantitativen Teils wurde der Fragebogen online oder in Papierform zugänglich gemacht.

Mit diesem Forschungsdesign wird den folgenden aufeinander aufbauenden Forschungsfragen nachgegangen: Welche Werte präferieren Lehrkräfte (a) für sich persönlich, (b) berufsbezogen und aus der (bayrischen) Verfassung abgeleitet, (c) bezüglich der Wertevermittlung an die Schüler*innen und (d) welcher Zusammenhang besteht mit personenbezogenen Variablen wie Geschlecht, Alter Unterrichtsfach etc.? Dem schließt sich die qualitativ ausgerichtete Forschungsfrage nach den Werthaltungen, also der Interpretation der präferierten Werte an (vgl. S. 95 ff.).

Das dem quantitativen Teil zu Grunde gelegte Wertekonstrukt entwickelt Felser aus einer Auseinandersetzung mit dem sozial- und gesellschaftswissenschaftlichen Terminologien des Wertebegriffs, empirischen Forschungen zum Wertewandel in der Bundesrepublik und der Bedeutung von Werten im Kontext Schule. Des Weiteren fließen rechtlich-administrative und (bildungs-)politische Rahmenbedingungen sowie die Bedeutung von Werten auf der Ebene der individuellen Professionalität in die Auseinandersetzung ein.

Zusammengenommen schärft dies den Blick Axel Felsers für den Wertebegriff in den Spannungsfeldern von Objektivität und Subjektivität, Allgemeingültigkeit und Kontextabhängigkeit sowie Orientierungsfunktion und Handlungsrelevanz (S. 32). Ebenso fokussiert er die Bedeutung der individuellen Varianz von Wertepräferenzen zwischen Lehrer*innen, obwohl diese als Berufsgruppe überwiegend dem liberalintellektuellen Milieu entstammen würden (S. 95). Schließlich beleuchtet er die Wertethematik angesichts der teilweise divergierenden sozialen Ansprüche von Kollegium, Schulleitung, Schulaufsicht und den Eltern und gesetzlichen sowie (bildungs-)politischen Anforderungen, mit denen sich Lehrkräfte als Vertreter*innen der Institution Schule gleichermaßen konfrontiert sehen.

Vor diesem theoretischen Hintergrund wurden für den quantitativen Teil der Studie folgende Instrumente verwendet, die eine eigene Weiterentwicklung und Faktorenanalyse erfuhren: Zur Analyse der persönlichen Werte von Lehrer*innen wurde der Portrait Values Questionnaire (PVQ) (Schmidt et al. 2007) ausgewählt, durch den individuelle Werttypen in Form von Porträts aus den Wertbereichen Macht, Leistung, Hedonismus, Stimulation, Selbstbestimmung, Universalismus, Benevolenz, Tradition, Sicherheit und Konformität generiert werden. Außerdem fand das Instrument „The Wall“ Verwendung, bei dem von den Studienteilnehmer*innen aus 47 Wertbegriffen neun Werte ausgewählt und in ein dreistufiges Ranking (wichtig, sehr wichtig, unverzichtbar) gebracht werden. Für die Analyse berufsbezogener Werte wurde zum einen das Instrument „The Wall“ in Bezug auf die Vermittlung der Werte an Schülerinnen und Schüler eingesetzt und zum anderen das selbst entwickelte und getestete Befragungsinstrument ,Werte im Kontext der eigenen Tätigkeit als Lehrkraft", welches aus den vier Skalen Engagement, Partizipation, Empathie und Sorgfalt/Ordnung besteht. Zur Erhebung der Persönlichkeitsstruktur wurde eine Kurzversion des Big-Five-Inventory (BFI-K) eingesetzt.

Als Ergebnis des quantitativen Teils konnte eine große Zustimmung zu den Wertebereichen Benevolenz, Selbstbestimmung und Universalismus auf persönlicher Ebene im PVQ herausgefunden werden, die damit für Felser ,,als eine Art Konsens“ anzusehen sind, was sich auch in anderen Studien zeige (S. 230). Jedoch weise die 
breite Streuung hinsichtlich der Zustimmung zu den anderen Wertebereichen (insbesondere Stimulation und Leistung, aber auch Macht) darauf hin, ,dass sich Wertpräferenzen innerhalb von Kollegien teilweise erheblich voneinander unterscheiden können“ (S. 231). Zu Recht diskutiert Felser die Frage, ob sozial erwünschtes Antwortverhalten hier eine besondere Rolle spielt und aus welchen Gründen die Begriffe als bedeutsam angesehen werden, also beispielsweise ob die Werte einen Ist- oder einen Soll-Zustand beschreiben (S. 231).

Auf beruflicher Ebene dokumentierte sich eine hohe Zustimmung zum Wertebereich Empathie/Fürsorge im Sinne des Eingehens auf die persönlichen Bedürfnisse der Schüler*innen und eine mittlere Präferenz für die Wertebereiche Partizipation von Schüler*innen (S. 231). Gleichzeitig werden mit Bezug auf Werte, die das Lernund Arbeitsverhalten betreffen, andere Präferenzen hinsichtlich der an Schüler*innen zu vermittelnde Werte als im persönlichen Leben genannt. Wenn Lehrer*innen eine Funktionsstelle innehaben, korreliert dies positiv mit den Wertebereichen Macht und Engagement (S. 233). Hingegen konnte kein Unterschied zwischen Lehrkräften an staatlichen Regelschulen und Schulen privater Trägerschaft festgestellt werden (S. 246).

Inferenzstatistisch bestätigt sich Felsers Ausgangsannahme, dass „die Wertepräferenzen in erster Linie mit den Persönlichkeitsmerkmalen der befragten Lehrkräfte korrelieren“ (S. 232). Ebenso zeigte sich, dass die Persönlichkeitsmerkmale deutlich mehr die Varianz zwischen den Wertorientierungen aufklären können als etwa die schulbezogenen Variablen (S. 233).

Die qualitative inhaltsanalytische Untersuchung bestätigt im Wesentlichen die Wertepräferenzen der quantitativen Studie. Darüber hinaus deute sich an, „dass die Lehrkräfte Werte für den Schulkontext in erster Linie im Sinne einer Vermittlung an bzw. Thematisierung mit den Schülerinnen und Schülern verstehen“ und Werte als Orientierung für das eigene Verhalten und Handeln anzusehen scheint ,eher zweitrangig zu sein bzw. zeigen sich in dieser Hinsicht große Unterschiede zwischen den Lehrkräften“" (S. 235).

Interessant sind auch Nebenergebnisse wie jene, dass die Zustimmung zum Wertebereich Heimat aus der Bayerischen Verfassung negativ ausfällt, was der Autor mit ,den aktuellen gesellschaftlichen Diskursen um Heterogenität, Migration und dem kontrovers diskutierten Selbstverständnis der eigenen Nation“ erklärt (S. 232) oder der stark positive Einfluss des Unterrichtsfachs Religion in Bezug auf den Wertebereich Tradition (S. 233). Bedeutsam scheinen darüber hinaus ethische Dilemmata, die sich in der qualitativen Untersuchung induktiv erschlossen haben: So wird es ,als schwierig empfunden, bestimmte Formen der Umsetzung von Natur- und Umweltbewusstsein, beispielsweise der Kauf von umweltbewussten Schulmaterialien, wie Heften oder Stiften, von der eigenen Person auf die Eltern zu übertragen“ (S. 207).

Was lässt sich aus der Studie festhalten? Unter dem Dach bestimmter kollektiv geteilter schulbezogener Werte zeigt sich Schule als Ort ethischen Pluralismus, in dem unterschiedliche Werthaltungen von Lehrer*innen aufeinandertreffen. Damit signalisieren die Ergebnisse Axel Felsers für das Feld der erziehungswissenschaftlichen Heterogenitätsforschung (vgl. Budde et al. 2015; Koller 2014) den Aspekt der Heterogenität von Werten und Werthaltungen im Lehrer*innenkollegium deutlicher in den Blick zu nehmen. Aus den Erkenntnissen seiner Studie zieht Felser ein 
wichtiges Resümee, das für alle Schulentwicklungsprozesse berücksichtigt werden sollte: „Die potenziell vielfältigen Interpretationsrichtungen innerhalb ein und desselben Wertebegriffs zeigen, dass die Lehrkräfte für sich selbst, aber auch innerhalb ihrer Kollegen Zeit brauchen, um diese Klärungsprozesse vorzunehmen“ (S. 247). Dies ernst genommen, kann die Bedeutung von Schule als bedeutsamer Ort demokratischer Kultur weiter gefestigt werden. Dann jedoch braucht es nicht nur einen Klärungsprozess über die vielfältige Interpretation von Wertebegriffen, sondern ein Bewusstsein für die Notwendigkeit kontinuierlicher deliberativer Prozesse im Lehrer*innenkollegium als Teil der Organisationskultur sowie schulischer Fortbildungen. Aus der Perspektive von Schüler*innen stellt sich die Frage, ob angesichts empirisch feststellbarer Praxen der Beschämung und Missachtung von Schüler*innen (Prengel 2012; Wertenbruch und Röttger-Rössler 2011) unterschiedliche Wertehaltungen ein ursächlicher Faktor für Konflikte sind und die Auseinandersetzung mit den eigenen und kollegial bestehenden Wertepräferenzen und Wertehaltungen ggf. einen Schlüssel für Professionalisierungsbewegungen im schulischen Feld darstellen.

Funding Open Access funding enabled and organized by Projekt DEAL.

Open Access Dieser Artikel wird unter der Creative Commons Namensnennung 4.0 International Lizenz veröffentlicht, welche die Nutzung, Vervielfältigung, Bearbeitung, Verbreitung und Wiedergabe in jeglichem Medium und Format erlaubt, sofern Sie den/die ursprünglichen Autor(en) und die Quelle ordnungsgemäß nennen, einen Link zur Creative Commons Lizenz beifügen und angeben, ob Änderungen vorgenommen wurden.

Die in diesem Artikel enthaltenen Bilder und sonstiges Drittmaterial unterliegen ebenfalls der genannten Creative Commons Lizenz, sofern sich aus der Abbildungslegende nichts anderes ergibt. Sofern das betreffende Material nicht unter der genannten Creative Commons Lizenz steht und die betreffende Handlung nicht nach gesetzlichen Vorschriften erlaubt ist, ist für die oben aufgeführten Weiterverwendungen des Materials die Einwilligung des jeweiligen Rechteinhabers einzuholen.

Weitere Details zur Lizenz entnehmen Sie bitte der Lizenzinformation auf http://creativecommons.org/ licenses/by/4.0/deed.de.

\section{Literatur}

Benner, D. (2009). Moralische Erziehung und Bildung der Moral. TOPOLOGIK, 6, 114-121.

Budde, J., Blasse, N., Bossen, A., \& Rißler, G. (Hrsg.). (2015). Heterogenitätsforschung. Weinheim, Basel: Beltz Juventa.

Heinzel, F., Prengel, A., Reitz, S., \& Winklhofer, U. (2017). Reckahner Reflexionen zur Ethik pädagogischer Beziehungen. Reckahn: Rochow Edition.

Höhn, E. (2003). Wandel der Werte und Erziehungsziele in Deutschland: Eine soziologisch-empirische Bestandsaufnahme der gegenwärtigen gesamtgesellschaftlichen Situation mit Schwerpunkt auf Schule und Familie im Kontext des 20. Jahrhunderts (1. Aufl.). Frankfurt a.M: Hänsel-Hohenhausen.

Koller, H.-C. (2014). Heterogenität - Zur Konjunktur eines pädagogischen Konzepts. In H.-C. Koller, R. Casale \& N. Ricken (Hrsg.), Heterogenität. Zur Konjunktur eines pädagogischen Konzepts. Paderborn: Ferdinand Schöningh.

Oser, F. (1998). Ethos - die Vermenschlichung des Erfolgs. Zur Psychologie der Berufsmoral von Lehrpersonen. Opladen: Leske + Budrich.

Prengel, A. (2012). Respekt und Missachtung: Interaktionen zwischen LehrerInnen und SchülerInnen. In S. Andresen \& W. Heitmeyer (Hrsg.), Zerstörerische Vorgänge (S. 178-194). Weinheim, Basel: Beltz Juventa. 
Schmidt, P., Bamberg, S., Davidov, E., Herrmann, J., \& Schwartz, S. (2007). Die Messung von Werten mit dem „Portraits Value Questionaire“. Zeitschrift für Sozialpsychologie, 38(4), 261-275.

Wertenbruch, M., \& Röttger-Rössler, B. (2011). Emotionsethnologische Untersuchungen zu Scham und Beschämung in der Schule. Zeitschrift für Erziehungswissenschaft, 14, 241-257. 